\title{
Mechanical analysis on cement sheath integrity under asymmetric load
}

\author{
Xiaoyu Zhang ${ }^{1,2} \cdot$ Lei Wang $^{1} \cdot{\text { Zhenhui } \mathrm{Bi}^{1} \cdot \text { Yintong Guo }}^{1} \cdot$ Chunhe Yang $^{1} \cdot$ Hanzhi Yang $^{3} \cdot$ Xin Chang $^{1}$
}

Received: 27 September 2021 / Accepted: 19 October 2021 / Published online: 29 October 2021

(c) The Author(s) 2021

\begin{abstract}
In large-scale multi-section hydraulic fracturing, the stress environment of wellbore is extreme complex, often causing the unbalanced stress distribution around the wellbore. That poses great challenges to the integrity of the sheath. In this paper, firstly, triaxial compression test and triaxial cyclic test are carried out at $130{ }^{\circ} \mathrm{C}$ to study the deformation characteristics of the cement under high temperature. Then based on that, an appropriate plastic mechanics model is established. Finally, the shakedown theory is applied to analyze the model and acquires a maximum cyclic loading under asymmetric stress. The result shows that (1) the well cement, with the increase of load, shows the plastic flow characteristics and can be regarded as an ideal elastic-plastic material under high temperature. (2) During the cyclic loading and unloading process, the "hysteresis loop" becomes denser, which indicates that the accumulation rate of plastic deformation is continuously declining. The main plastic strain appears in the phase of the first loading. (3) The external pressure $P_{z}$ plays a positive role in the deformation control of the sheath. With the growth of $P_{z}$, the maximum cyclic loading $P_{\max }$ will also increase. (4) Asymmetric stress distribution can significantly affect the bearing capacity of the sheath. If stress difference coefficient $\lambda=0.3$, the $P_{\max }$ tends to decrease nearly by $50 \%$. With the growth of $\lambda$, the negative influence of stress asymmetry reduces gradually. High external pressure is beneficial to reduce the negative impact of the asymmetry. With the growth of $\lambda$, the benefit tends to enhance. (5) In engineering practice, if the geology around wellhole showcases the strong asymmetry (the value of $\lambda$ is large), some steps need to be adopted to reduce the stress concentration.
\end{abstract}

Keywords Deep shale gas $\cdot$ Cement sheath $\cdot$ Shakedown theory $\cdot$ Asymmetry

\section{Introduction}

Compared with conventional oil and gas field development, large-scale multi-section hydraulic fracturing can have a special impact on the wellbore integrity. This is due to the extreme complex stress environment of wellbore. First, the downhole geology conditions are usually complex and unpredictable, the tectonic stress of the formation to the wellbore is usually not uniform, and the stress difference always exists. Especially, when there are faults or other large

Yintong Guo

ytguo@whrsm.ac.cn

1 State Key Laboratory of Geomechanics and Geotechnical Engineering, Institute of Rock and Soil Mechanics, Chinese Academy of Sciences, Wuhan 430071, Hubei, China

2 University of Chinese Academy of Sciences, Beijing 100049, China

3 Chongqing University, Chongqing 400030, China geological structures around the wellbore, the stress distribution will suddenly change and show strong asymmetry, which greatly challenges the integrity of the sheath.

In recent years, with the increasing prominence of oil and gas leakage caused by sealing failure, scholars in the whole world have carried out a series of researches on the rock properties, analytical theory, numerical simulation, and operation apparatus of cementing. A number of results have been obtained.

Triaxial compression and cyclic loading/unloading tests were designed for cement at room temperature and elevated temperatures of $95^{\circ} \mathrm{C}$ and $130{ }^{\circ} \mathrm{C}$. The results demonstrate that the temperature higher, the cement cumulative residual strain became larger, and there was a significant "rebound hysteresis" in the unloading curve (Zhou et al., 2019). The law of mechanical damage-induced deformation of microsphere low-density cement sheaths was studied based on triaxial cyclic loading. The experimental results indicate that temperature has the greatest impact on the mechanical 
integrity of micro-sphere low-density cement sheaths. The selection of micro-spheres and the mechanical modification of cement sheaths are key contributors to the integrity of micro-sphere low-density cement sheaths (Li et al., 2017). Considering the radial nonuniform temperature change, a new thermal stress model for a casing-cement sheath-formation combined system for geothermal wells during fracturing based on elastic mechanics and thermodynamics theory is proposed. According to the analysis, the radial and axial tensile thermal stresses are both obviously larger than tangential tensile thermal stress. The maximum radial and axial thermal stresses always occur at the casing interface even if the location of the maximum tangential thermal stress varies. Generally, thermal stresses are more likely to induce radial and axial micro-cracks in the cement sheath, and the cement sheath will fail more easily at the casing interface in fracturing geothermal wells (Xu et al., 2018b). The shear strength and hydraulic permeability of the interface between well cement and casing were investigated based on a triaxial direct shear apparatus under controlled stress conditions with fluid flow measurements along the interface. The results indicate the shear strength of the interface is provided by friction and the cement-casing interface is not a significant leakage pathway for gas (Welch et al., 2020). The influence of interface defects on shear strength at cement-interlayer interface (CII) and fluid channeling was discussed. Then, a mathematical model between missing amount of mud cake ring and shear strength at CII was developed, which reveals that interface defects drastically affect fluid channeling at CII (Gu et al., 2012). A new mechanical model of cement sheath for fracturing wells, coupling pressure, and thermal loads was proposed. In this model, the failure modes of debonding, radial cracking, disking, and shear failure have been taken into consideration. Results show that the decreased wellbore temperature will produce significant triaxial tensile stress and induce cement failure of de-bonding, radial cracking, and disking. The increased casing pressure will significantly lower the risk of de-bonding but also aggravate radial cracking and shear failure (Xu et al., 2018a). Based on the interface bonding mechanism of "geological body-engineering body" binary media materials, the control indexes affecting the bonding performance of rock-concrete interface were explored, and the theoretical characterization of the interface bonding strength of binary media was constructed. Then, a bond strength freeze-thaw degradation model of rock-concrete interface was proposed through the internal cognition of the degradation process of the interface bonding performance under freeze-thaw (Shen et al., 2020). The possibility of formation damage reduction experimentally was investigated during leak-off and flow back processes by adding surfactants to the fracturing fluid. The results indicate that adding soloterra 983 surfactants to the saline reservoir brine can reduce the pressure drop during leak-off and flow back processes, therefore, decreasing the possibility of aqueous phase trapping and formation damage in the Montney tight core plugs (Yarveicy et al., 2018). An analytical approach based on the theory of thermo-poroelasticity was adopted to predict the possibility of cement sheath failure in deep structures. The results obtained proclaim that a thicker cement can withstand a higher load applied by the formations and protect the casing against a significant collapse pressure. The temperature was also found as a significant contributor to increasing the pressure applied by the formation and casing on the cement caused by pore fluid and steel expansions (Gholami et al., 2016). To analyze the effect of the thermal expansion annulus pressure on the cement sheath mechanical integrity, the cement sheath safety factor calculation model with thermal expansion annulus pressure considered was established. The results show that the cement sheath safety factor reaches maximum when the thermal expansion coefficients of casing, cement sheath, and formation are equal to each other (Z. Zhang and Wang, 2017). A novel elastic-plastic model incorporating the effects of drilling, cementing, and dramatic temperature change of the well system during the hydraulic fracturing was established and the failure mechanism of cement sheath was fully revealed. The results illustrate that the tensile failure is more likely to occur at the inner face of the cement sheath, which turns out to be more severe as the true vertical depth decreases. The density of cement slurry is in favor of decreasing the tensile stress in the cement sheath. (Zhao et al., 2019). How the integrity of the cement and casing is affected during these operations is studied in casing and cement by finite element analysis (FEA). Simulation results revealed that a poor centralization that contributes to the formation of drilling fluid voids in the cement will induce casing failure during hydraulic fracturing operations (Restrepo et al., 2020). A model of casing-cement sheath formation combination under creep formation conditions was developed. The effects of the insitu stress (ISS) and cement sheath's elastic parameters on the seal integrity of the cement sheath were studied in the salt rock layer. The creep of the salt rock increased the stress acting on the cement sheath over time and caused shear failure of the cement sheath. A larger uniform horizontal ISS led to a shorter period for the shear failure of the cement sheath, larger equivalent plastic strain (PEEQ), and larger yield area (Yang et al., 2021). Based on the scientific conception of Mud Cake to Agglomerated Cake (MTA), the integrated solidification and cementation of cement-formation interface were studied by mechanical evaluation, microstructure analysis, and fluid dissolution test. The research shows the bond strength of cement-formation interface increased by $37.81 \% \sim 1606.31 \%$ with MTA method. (Jun and Wenzheng 2010). An analytical model considering the well-completion steps was established for the purpose of studying the integrity of cement sheath as a two-layer 
casing-cement system. The results show that the tensile failure is more likely to occur at the inner face of the cement sheath in the intermediate casing. The casing thickness is in favor of decreasing the tensile stress in the cement sheath. However, on the contrary, increasing the thickness and elastic modulus of the cement sheath may aggravate the risk of tensile failure in the cement sheath while reducing the costs (Liu et al., 2018). Based on the characteristics of the cementing process in shale reservoir horizontal wells and given the APB (Annular Pressure Buildup) effect in conventional wells, both physical models and finite element models were established. Many factors, such as the void angles, temperature change, magnitude of the inner pressure, and in-situ stress were investigated. The results show that (1) a high pumping pressure was needed in a high geo-stress shale block to fracture the formation, inducing a higher internal pressure in the casing. Last but not the least, (2) under the combined effect of high internal pressure and non-uniform external support, the downhole casing has a high risk of deformation. (Yan et al., 2017). In this paper, an analytical model is derived to predict the Maximum Allowable Fracture Pressure (MPFP) caused by radial cracks by taking into account the weakening of cement sheath and the reduced support of formation rock. It shows that improving cement strength fails to effectively increase MPFP. The MPFP should be enhanced by increasing the thickness of the cement sheath using large wellbore size and improving the cement placement efficiency. This work also shows that cement sheath failure will occur before casing failure occurs in the hydraulic fracturing process. Therefore, integrity of cement sheath should be considered in the hydraulic pressure design. (Guo et al., 2018). Three-point bending test of bi-material beams was used to study tensile failure at cement-steel, cement-cement, and cement-sandstone interfaces. The tests revealed that cement-steel interfaces were the weakest ones, while cement-cement interfaces were the second weakest. All bi-material beams had lower flexural strength than monolith cement beams (Lavrov et al., 2017). A new device of bypass cementing device, along with the indispensable accessories, was proposed and designed. The shear deformation degrees before and after using the new device were compared by numerical method. Simulation results showed that after using the bypass cementing device, the reduction of casing inner diameter after fault slipping was reduced by about $63.75-75.6 \%$, which couldeffectively ensure the casing integrity (Xiet al., 2021). The large-scale sealing evaluation equipment to assess the failure mechanisms of cement sheaths under cyclic loading is present. The failure mechanisms of the sealing integrity of the cement sheath were obtained by combining tests and the finite element calculations. (Zeng et al., 2019). A new way of evaluating and understanding the behavior of micro annulus sealing function of the cement sheath-casing interface by taking the high-resolution optical device as a function of cement curing age and pipe lengths is demonstrated. The technical benefit of this novel experiment is to quantify the primary functional purpose of the cement which is supporting the casing and to seal the annulus to the flow of fluid (Al Ramis et al., 2020). An analytical model of the stress, displacement, and interaction of casing-cement sheath-surrounding rocks subjected to inner casing pressure and outer radial far-field insitu stress and debonding of cementing surfaces were derived with due consideration of varied casing pressure, temperature, and pore pressure due to cyclic injection and production. The results indicate that the variation of temperature, pore pressure and far-field in-situ stress are key factors affecting the stress $\&$ deformation of cement sheath and micro-annulus. For cement with large elasticity modulus and small Poisson's ratio, plastic deformation and microannulus are prone to forming (H. Zhang et al., 2017).

From the discussion above, although the research on the sheath from many aspects is relatively in-depth, however, previous studies are mostly based on linear elasticity. At the same time, the researches on complex periodic loads, especially the asymmetric case, are comparatively insufficient. Consequently, taking the ideal elastoplasticity into account, this paper used shakedown theory to clarify the influence of the asymmetric loading on the sheath integrity. This research can provide guidance for engineers to design projects onsite and is useful for ensuring long-term, safe and efficient development of shale gas resources.

\section{Mechanical properties of oil well cement under high temperature}

\section{Sample preparation}

First of all, the G-grade high sulfate-resistant oil well cement, liquid fluid loss agent DZJ-Y, silica fume (200 mesh), and water were mixed into cement slurry based on the ratio of 1:0.04:0.35:0.42. Then, the mixture was formed in a specified size in a cylindrical mold and cured in waterbath heating at the temperature of $130{ }^{\circ} \mathrm{C}$ and pressure of 20.7 MPa for 3 days. This curing condition is to simulate the environment of hydration, condensation, and hardening of cement in the deep stratum. After that, samples were preserved in a water bath at $27^{\circ} \mathrm{C} \pm 3{ }^{\circ} \mathrm{C}$. The sample parameters are shown in Table 1.

Before the tests, some measures must be taken as follows: (1) the unevenness error at both ends of the sample shall not be greater than $0.05 \mathrm{~mm}$; (2) the diameter error along the height of the sample shall not be greater than $0.3 \mathrm{~mm}$; (3) the end section shall be perpendicular to the axis of the sample, and the maximum deviation shall not exceed $0.25^{\circ}$. In this way, samples meeting the requirements SL/T 
Table 1 Parameters of samples for mechanical tests

\begin{tabular}{lllll}
\hline Sample number & Diameter $(\mathrm{mm})$ & Height $(\mathrm{mm})$ & $\begin{array}{l}\text { Confining pressure } \\
\sigma_{3}(\mathrm{MPa})\end{array}$ & $\begin{array}{l}\text { Tem- } \\
\text { perature } \\
\left({ }^{\circ} \mathrm{C}\right)\end{array}$ \\
\hline $2-6$ & 50.07 & 99.84 & 15 & 130 \\
$2-7$ & 50.02 & 99.85 & 15 & 130 \\
\hline
\end{tabular}

264-2020, Specification for rock test of water conservancy and Hydropower Engineering[S] of the specifications are ready to be tested.

\section{Analysis of experimental results}

\section{Triaxial compression test at $130^{\circ} \mathrm{C}$}

The curve soon begins to deviate from the straight portion, and then the sample undergoes plastic deformation. With the increase of load, we can see the continuous accumulation of plastic deformation. Similarly, the curve shows slow growth and tends to be flat, showing plastic flow characteristics.

The peak deviator stress of the sample is $39.50 \mathrm{MPa}$, the peak strain is $4.41 \%$, and the elastic modulus is $3.89 \mathrm{GPa}$. Poisson's ratio is 0.071 . At $130{ }^{\circ} \mathrm{C}$, the compressive strength of the sample maintains high, just with a small decline. After the test, the sample is shorter than before, and few changes occur in the lateral and no manifest macro cracks on the surface. A few vertical micro-cracks can be observed on the upper-end face. Consequently, cement at high temperature can be considered as a category of ideal plastic material. The stress-strain curve and failure mode are shown in Fig. 1.

\section{High temperature triaxial cyclic test}

The upper and lower limits of cyclic load are set to $28 \mathrm{MPa}$ and $1 \mathrm{MPa}$, respectively. The upper limit load is $70 \%$ of the peak value of the triaxial compression test. The number of cycles is 20 , and the cyclic loading rate is $0.25 \mathrm{MPa} / \mathrm{s}$.
The deviator stress-strain curve first grows in an approximately straight line segment during the initial loading process at the temperature of $130{ }^{\circ} \mathrm{C}$. When the deviator stress reaches about $12.20 \mathrm{MPa}$, it starts to deviate from the straight line, suggesting plastic strain occurs. After the load reaches about $40 \mathrm{MPa}$, the unloading starts. The curve is approximately straight line, and slightly convex downward. The "hysteretic loop" formed between the unloading and reloading curves is in the shape of a thin strip. As the process progresses, new plastic strains, more and more slightly, continue to be generated. The width of the "hysteresis loop" continuously is reduced, in other words, "hysteresis loop" becomes denser, implying that the accumulation rate of plastic deformation is continuously declining. In addition, it is worth noting that the main plastic strain appears in the course of the first loading. The height of the sample was significantly shortened (Fig. 2), and no obvious macroscopic cracks are found, indicating that the sample shows mainly axial compression deformation and less lateral expansion.

When the temperature is $130{ }^{\circ} \mathrm{C}$, the stress-strain curve first grows in an approximately straight line during the initial loading process. When the deviatoric stress increases to $12.20 \mathrm{MPa}$, it begins to deviate from the straight line, indicating the occurrence of plastic strain. When the load reaches about $40 \mathrm{MPa}$, the unloading curve starts, approximately straight, slightly protrudes downward. When unloading to about $1 \mathrm{MPa}$, it will reverse to load. The "hysteresis loop" formed between unloading and reloading curves is thin strip. In pace with the process, new plastic strains occur more and more slightly. The width of the "hysteresis loop"
Fig. 1 Triaxial compression test at $130{ }^{\circ} \mathrm{C}: \mathbf{a}$ stress-strain curve of 2-6; b sample 2-6 morphology after test

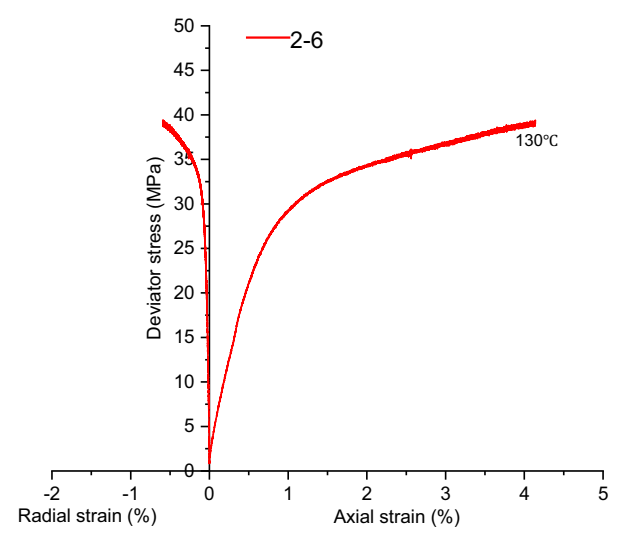

(a)

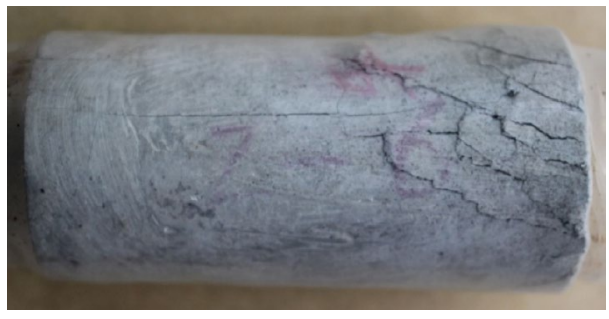

(b) 
Fig. 2 Cyclic loading test at $130^{\circ} \mathrm{C}$ : a stress-strain curve of 2-7; b sample 2-7 morphology after test

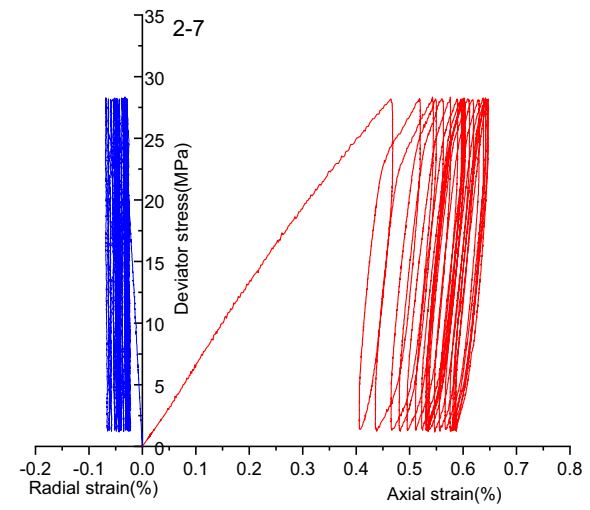

(a)

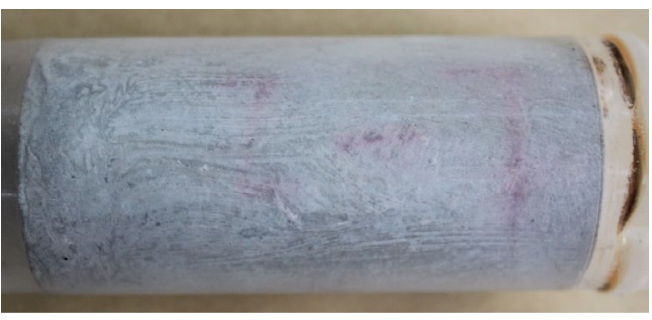

(b) decreases, which means that the cumulative rate of plastic deformation is continuously declining. What is noteworthy is that the main plastic strain occurs during the first loading course. The sample is obviously shortened after the test (Fig. 2), and no obvious macro cracks are found, indicating that the axial deformation is the primary and lateral is secondary.

\section{Mechanical model and analysis method}

\section{Mechanical model of cement sheath}

The sheath is an annular structure made of cement, and its internal and external diameter are generally fixed, with an inner diameter of $139.7 \mathrm{~mm}$ and an outer diameter of $233.5 \mathrm{~mm}$ in engineering practice The ratio of wall thickness to outer diameter is greater than 0.05 , which can be regarded as a thick-walled cylinder. The simplified diagram of the mechanical model of the cement sheath is shown in Fig. 3. Before the analysis, the mechanical model of the sheath constructed in this paper is based on the following assumptions:

1) Ideally, all materials constituting the sheath are stirred evenly, and there are no micro-cracks and bedding after hardening. Therefore, the cement stone can be seen as a kind of isotropic material.

2) According to mechanical properties of oil well cement under high temperature in the Sect. 2, cement can be regarded as an ideal elastic-plastic material.

3) Before the sheath bears the load, it is intact. The cementing quality is good, and both formation-sheath and sheath-casing interfaces maintain deformation compatibility before yielding of the sheath occurs.

4) When the sheath begins to produce plastic deformation and it continues to accumulate, the stress state at a certain point will satisfy the Mohr-Coulomb criterion. At this time, we think the function of the sheath is invalid.
That is to say, the sheath follows the Mohr-Coulomb yielding criterion (1-a), (1-b), (1-a), and (1-b) is loading condition and unloading yield condition separately.

$$
\begin{aligned}
& \sigma_{r}=\sigma_{\theta} \operatorname{tg}^{2}\left(45^{\circ}+\frac{\phi}{2}\right)-2 \operatorname{ctg}\left(45^{\circ}+\frac{\phi}{2}\right) \\
& \sigma_{\theta}=\sigma_{r} \operatorname{tg}^{2}\left(45^{\circ}+\frac{\phi}{2}\right)-2 \operatorname{ctg}\left(45^{\circ}+\frac{\phi}{2}\right)
\end{aligned}
$$

$c$ refers to the cohesive force of the cement, $\mathrm{MPa}$; and $\varphi$ means the internal friction angle, ${ }^{\circ} ; \sigma_{r}$ and $\sigma_{\theta}$ represent radial stress and circumferential stress respectively, MPa. For the convenience of equation derivation, let:

$K=\operatorname{tg}^{2}\left(45^{\circ}+\frac{\phi}{2}\right)$

$B=-2 \operatorname{ctg}\left(45^{\circ}+\frac{\phi}{2}\right)$

The stress diagram of the sheath in horizontal section is shown in Fig. 3, $P_{Z}$ and $\lambda P_{Z}$ are vertical pressure and horizontal pressure, respectively, MPa. The reflecting degree of load asymmetry is stress difference coefficient. The cyclic inter pressure on cement sheath is $P_{i}, \mathrm{MPa}$, and the internal and external radii are $a$ and $b$ respectively, mm.

\section{Analysis method}

The sheath downhole is always in a periodic asymmetric loading, so there are few relevant theories and methods involving can be applied in the petroleum field. However, as the sheath is essentially a cement structure bearing complex stress, the shakedown theory as the limit analysis method commonly used in civil engineering can be adopted.

Shakedown was first proposed by plastic mechanist Prager. It is used to describe the adaptability of elastic-plastic media 
Fig. 3 Schematic diagram of cement sheath model

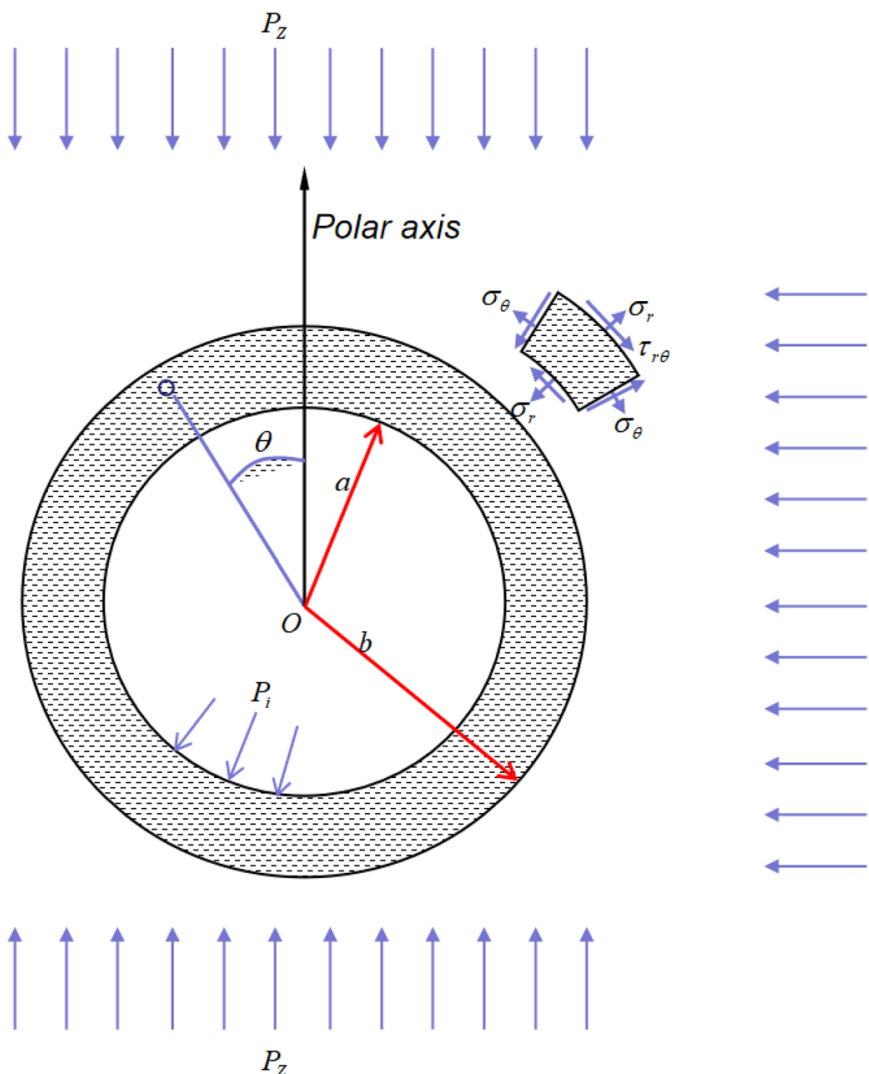

subjected to cycles of load under the plastic deformation. Shakedown analysis focuses on the plastic behavior of structures under repeated loading. If structures undergo a given range of varying loads, there may be two outcomes: (1) after cyclic loading is repeated several times, plastic deformation tends to be stable and gradually forms a favorable residue-stress distribution. The favorable residue-stress distribution will play a positive role in the subsequent stress state of the sheath and make the plastic deformation no longer accumulate. That means that if the sheath undergoes cyclic loading in a calculated domain, it will be regarded as an elastomer after the first stress cycle. The subsequent performance of the structure is an elastic one. At that time, the structure is stable; (2) the plastic deformation increases or reverses continuously under the action of repeated load. The structure will fail to work. According to the shakedown theory, we can find a cyclic loading domain in which the structure can operate normally. On the contrary, if the load exceeds this range, the plastic deformation will continue to develop and accumulate, resulting in cumulative plastic deformation failure or alternating plastic failure.

The study in this paper is based on the lower bound shakedown theory called Melan's theorem. It is adopted for the analysis of cement sheath and expressed as follows: if a self-equilibrium stress field independent of time can be built and the elastic stress field generated by any external load within the given load range is provided, the structure is stable. The equation is shown as follows:

$\max : v$

s.t.f $\left[v \sigma_{i j}^{E}(x, t)+\rho_{i j}(x)\right] \leq 0 \forall x \in V$

$\rho_{i j, j}(x)=0 \forall x \in V$

$\rho_{i j, j}(x) n_{j}=0 \forall x \in S_{\sigma}$

where $\nu$ is the load multiplier; $\sigma^{E}{ }_{i j}(x, t)$ refers to the elastic stress field under any loading path in any loading domain within a given load; $\rho_{i j}$ means the self-balancing residual stress field; and $f$ is the yield function (Neal B. G., 1956).

The key to solving the problem by using Melan theorem is to build the self-equilibrium stress field independent of time. As the geometric structure of cement sheath is special, it is easier to get the analytical solution of stress distribution. Therefore, the direct construction residual stress method (DCRS) (Zarka J et al., 1979) is used to obtain the self-balanced stress field, that is, the stress-strain space in the structure can be directly obtained by elastic analysis and the first elastic-plastic cycle. Therefore, the direct structural residual stress (DSRS) method can take the convenience and accuracy of calculation in engineering into consideration at the same time.

According to the above discussion, there is some elastic-plastic material subjected to volume force $F$ and surface 
force $T$, and the displacement boundary is given. Given virtual residual stress $\bar{\sigma}_{i j}^{r}$ dependent on time $t$, the complete elastic solution $\bar{\sigma}_{i j}^{E}$, anywhere in the object, meets

$f\left(\bar{\sigma}_{i j}^{r}+\bar{\sigma}_{i j}^{E}\right) \leq 0$

Then the structure is in shakedown state. That indicates the structure will no longer produce plastic deformation after a certain cycle, and the stress generated by the loading and residual stress are still in yield surface after superposition. (Zarka J et al., 1979).

\section{Elastoplastic solution of the sheath under asymmetric load}

\section{Elastic solution of cement sheath stress under asymmetric load}

When the inter-pressure $P_{i}$ of the sheath is small, the whole cement sheath is in an elastic state. According to elasticity mechanics, the stress components can be expressed by stress function:

$$
\begin{aligned}
\varphi= & \frac{1}{4}\left[A_{1} r^{2}+A_{2}+\left(A_{3} r^{2}+A_{4}\right) \ln r\right. \\
& \left.+\left(A_{5} r^{4}+A_{6} r^{2}+A_{7}+\frac{A_{8}}{r^{2}}\right) \cos 2 \theta\right]
\end{aligned}
$$

where $A_{i}(1<i<8)$ is the undetermined constant.

According to the stress condition of sheath in the stratum, at the inner diameter,

$r=a\left\{\begin{array}{l}\sigma_{r}=P_{i} \\ \sigma_{\theta}=0 \\ \tau_{r \theta}=0\end{array}\right.$

At the external diameter,

$r=b\left\{\begin{array}{l}\theta=0, \sigma_{r}=P_{z} \sigma_{\theta}=0 \tau_{r \theta}=0 \\ \theta=\frac{\pi}{2}, \sigma_{r}=0 \sigma_{r}=\lambda P_{z} \tau_{r \theta}=0\end{array}\right.$

The linear equations about $A_{i}$ can be obtained by formula (1-a), (5), (6), (7), and (8). They can be written in matrix form (9). Because $A_{2}$ the constant term in the function is 0 after partial derivation, it does not affect the stress field according to (5) and (6).

$\left(\begin{array}{l}A_{1} \\ A_{3} \\ A_{4} \\ A_{5} \\ A_{6} \\ A_{7} \\ A_{8}\end{array}\right)=\left(\begin{array}{ccccccc}\frac{1}{2} & \frac{1+2 L n a}{4} & \frac{a^{-2}}{4} & 0 & -\frac{\cos 2 \theta}{2} & -a^{-2} \cos 2 \theta & -\frac{3 a^{-4} \cos 2 \theta}{2} \\ \frac{1}{2} & \frac{3+2 L n a}{4} & \frac{-a^{-2}}{4} & 3 a^{2} \cos 2 \theta & \frac{\cos 2 \theta}{2} & 0 & \frac{3 a^{-4} \cos 2 \theta}{2} \\ 0 & 0 & 0 & \frac{3 a^{2}}{2} & \frac{1}{2} & -\frac{a^{-2}}{2} & -\frac{3 a^{-4}}{2} \\ \frac{1}{2} & \frac{1+2 L n b}{4} & \frac{b^{-2}}{4} & 0 & -\frac{1}{2} & -b^{-2} & -\frac{3 b^{-4}}{2} \\ \frac{1}{2} & \frac{3+2 L n b}{4} & \frac{-b^{-2}}{4} & 3 b^{2} & \frac{1}{2} & 0 & \frac{3 b^{-4}}{2} \\ \frac{1}{2} & \frac{1+2 L n b}{4} & \frac{b^{-2}}{4} & 0 & \frac{1}{2} & b^{-2} & \frac{3 b^{-4}}{2} \\ \frac{1}{2} & \frac{3+2 L n b}{4} & \frac{-b^{-2}}{4} & -3 b^{2} & -\frac{1}{2} & 0 & -\frac{3 b^{-4}}{2}\end{array}\right)^{-1}\left(\begin{array}{c}P_{i} \\ 0 \\ 0 \\ P_{Z} \\ 0 \\ \lambda P_{Z} \\ 0\end{array}\right)$

$$
\left.\begin{array}{rl}
\sigma_{r} & =\frac{1}{r} \frac{\partial \varphi}{\partial r}+\frac{1}{r^{2}} \frac{\partial^{2} \varphi}{\partial \theta^{2}} \\
\sigma_{\theta} & =\frac{\partial^{2} \varphi}{\partial r^{2}} \\
\tau_{r \theta} & =-\frac{\partial}{\partial r}\left(\frac{1}{r} \frac{\partial \varphi}{\partial \theta}\right)
\end{array}\right\}
$$

Predecessors mostly use Lame's formula for elastic analysis under asymmetric loads, which led to large errors in the calculation results. In this paper, a trial method is used to find a stress function with certain accuracy under the premise that the deformation compatibility equation is naturally satisfied, which can be expressed as,
Each undetermined coefficient can be determined by the formula (9). Obviously, the solution process will be very complex. However, for the sheath, the size of its internal and external diameter does not change at will, and its internal and external diameter is fixed according to the standardized operation, that is, they can be regarded as known quantities. Therefore, substituting $a=139.7 \mathrm{~mm}$ and $b=233.5 \mathrm{~mm}$ into the matrix (9) can abate the calculation difficulty without changing the accuracy. After that, the undetermined coefficients are obtained. Obviously, they are only related to the internal pressure $P_{i}$, external pressure $P_{z}$ and pressure difference coefficient $\lambda$.

The stress field of cement sheath under asymmetric load is 
$\left.\begin{array}{rl}\sigma_{r}^{e} & =\frac{1}{2} A_{1}+\frac{1}{4} A_{3}+\frac{1}{4} A_{4} r^{-2}+\frac{1}{2} A_{3} \ln r-\cos 2 \theta\left(\frac{1}{2} A_{6}+A_{7} r^{-2}+\frac{3}{2} A_{8} r^{-4}\right) \\ \sigma_{\theta}^{e} & =\frac{1}{2} A_{1}+\frac{3}{4} A_{3}-\frac{1}{4} A_{4} r^{-2}+\frac{1}{2} A_{3} \ln r+\cos 2 \theta\left(\frac{1}{2} A_{6}+3 A_{5} r^{2}+\frac{3}{2} A_{8} r^{-4}\right) \\ \tau_{r \theta}^{e} & =\sin 2 \theta\left(\frac{1}{2} A_{6}+\frac{3}{2} A_{5} r^{2}-\frac{3}{2} A_{8} r^{-4}-\frac{1}{2} A_{7} r^{-2}\right)\end{array}\right\}$

The coefficients $A_{i}$ are as follows,

$\left[\begin{array}{c}A_{1} \\ A_{3} \\ A_{4} \\ A_{5} \\ A_{6} \\ A_{7}\end{array}\right]=\mu(a, b)\left[\begin{array}{c}P_{i} \\ P_{z} \\ \lambda P_{z}\end{array}\right]$

The parameter matrix $\mu(a, b)$ is determined by the size of the sheath, which is obtained by substituting $a=139.7 \mathrm{~mm}$ and $b=233.5 \mathrm{~mm}$ :

$\mu(a, b)=\left[\begin{array}{c}12.03+105.87 \cos 2 \theta \\ -1.96-16.50 \cos 2 \theta \\ (-5.75-30.07 \cos 2 \theta) 10^{-5} \\ 7.2 \times 10^{-6} \\ -2.11 \\ 1.08 \times 10^{-5} \\ -2.41 \times 10^{7}\end{array}\right.$
$f\left(P_{i}, P_{Z}, \lambda, \theta\right)=\sigma_{1}^{e}-K \sigma_{2}^{e}-B=0$

Also meet,

$\frac{\partial f\left(P_{i}, P_{Z}, \lambda, \theta\right)}{\partial \theta}=0$

Namely,

$\frac{\partial \sigma_{1}}{\partial \theta}-K \frac{\partial \sigma_{2}}{\partial \theta}=0$

According to formula (11), (12), (13), and (16), the orientation $\theta(\lambda)$ determined by $\lambda$ is of the first yield point at the
Obviously, according to the above formula (12), different from the symmetrical situation, the elasticity stress distribution is consistent with the azimuth $\theta$, so the points with the same stress are not on the same circumference. With the increasing internal pressure $P_{i}$, the points on the inner wall do not yield at the same time. A certain point or some points on the inner wall of the cement sheath yield first, rather than the points on the inner wall yield at the same time. The principal stress of the first yield points on the inner wall meets the yield formula (1-a), and the elastic limit pressure $P_{e}$ of the sheath ignoring some small and higher-order terms can be obtained.

The principal stress $\sigma_{1}{ }^{e}$ and $\sigma_{2 \theta}{ }^{e}, \mathrm{MPa}$, in the inner diameter, $r=a$, is,

$$
\left.\begin{array}{l}
\sigma_{1}^{e}=\frac{\sigma_{r}+\sigma_{\theta}}{2}+\sqrt{\left(\frac{\sigma_{r}-\sigma_{\theta}}{2}\right)^{2}+\tau_{r \theta}^{2}} \\
\sigma_{2}^{e}=\frac{\sigma_{r}+\sigma_{\theta}}{2}-\sqrt{\left(\frac{\sigma_{r}-\sigma_{\theta}}{2}\right)^{2}+\tau_{r \theta}^{2}}
\end{array}\right\}
$$

The yield point at the inner shall meet the Mohr-Coulomb law (1-a) inner diameter and the elastic limit internal pressure $P_{e}$ of the sheath can be obtained.

\section{Plastic solution of cement sheath stress under asymmetric load}

\section{Stress field in plastic zone}

When $P_{i}>P_{e}$, local plastic deformation occurs, and part of the cement sheath reaches the plastic state and the other is still in the elastic state. Obviously, the formula (7) still works. In the plane strain problem, for the sheath under asymmetric load, the stress field in the plastic zone should satisfy the equilibrium Eq. (17) in polar coordinates system,

$\left.\begin{array}{l}\frac{\partial \sigma_{r}}{\partial r}+\frac{1}{r} \frac{\partial \tau_{r \theta}}{\partial \theta}+\frac{1}{r}\left(\sigma_{r}-\sigma_{\theta}\right)=0 \\ \frac{1}{r} \frac{\partial \sigma_{\theta}}{\partial \theta}+\frac{\partial \tau_{r \theta}}{\partial r}+\frac{2 \tau_{r \theta}}{r}=0\end{array}\right\}$

After Eq. (1- $a$ ) and (7) into (17) is substituted (Sun and Lu, 2007; Yuxiang et al., 2012), the approximate stress distribution in the plastic zone $(a \leq r \leq \rho)$ is obtained, where $\rho$ is the yield diameter vector. 
$\left.\begin{array}{l}\sigma_{p_{r}}=-\left(-P_{i}+\frac{B}{1-K}\right)\left(\frac{r}{a}\right)^{\frac{1-K}{K}}+\frac{B}{1-K} \\ \sigma_{p_{\theta}}=-\frac{1}{K}\left(-P_{i}+\frac{B}{1-K}\right)\left(\frac{r}{a}\right)^{\frac{1-K}{K}}+\frac{B}{1-K}\end{array}\right\}$

The radial and normal pressures between the elastic and plastic zones can be derived from the above formula (17)

$\left.\begin{array}{l}P_{r}=\left(-P_{i}+\frac{B}{1-K}\right)\left(\frac{\rho}{a}\right)^{\frac{1-K}{K}}+\frac{B}{1-K} \\ P_{\theta}=\frac{1}{K}\left(-P_{i}+\frac{B}{1-K}\right)\left(\frac{\rho}{a}\right)^{\frac{1-K}{K}}+\frac{B}{1-K}\end{array}\right\}$

\section{Stress field in elastic zone}

Unlike the case of symmetric loading, it is clear that the yield vector diameter here is not a fixed value when internal and external pressures are definite, but changes with the $\theta$, that is, the entire elastic or plastic zone is not annular after deformation. Similarly, the stress field in the elastic zone can be expressed according to formula (10)

\section{Shakedown analysis of the sheath under asymmetric load}

\section{Constructing self-equilibrium stress field}

The residual stress field in the structure is directly obtained based on the elastic analysis and the first elastic-plastic cycle. If internal pressure $P_{i}\left(P_{i} \geq P_{e}\right)$ is applied to the cement sheath first, then the cement sheath will yield locally. After pressure $P_{u}$ is removed, the stress distribution at this time is the residual stress field, that is, the self-equilibrium stress field

$\left.\begin{array}{l}\sigma_{r}^{r}=\sigma_{r}^{p}\left(P_{i}, P_{z}\right)-\sigma_{1}^{e}\left(-P_{u}, P_{z}\right) \\ \sigma_{\theta}^{r}=\sigma_{\theta}^{p}\left(P_{i}, P_{z}\right)-\sigma_{2}^{e}\left(-P_{u}, P_{z}\right)\end{array}\right\}$

\section{Determining the allowable internal pressure in the sheath}

When reverse yielding occurs, according to formulas (20)

$\left.\begin{array}{rl}\sigma_{r}^{e} & =\frac{1}{2} A_{1 e}+\frac{1}{4}\left(A_{3 e}+A_{4 e} r^{-2}\right)+\frac{1}{2} A_{3 e} \ln r-\cos 2 \theta\left(\frac{1}{2} A_{6 e}+\frac{3}{2} A_{8 e} r^{-4}+A_{7 e} r^{-2}\right) \\ \sigma_{\theta}^{e} & =\frac{1}{2} A_{1 e}+\frac{3}{4} A_{3 e}-\frac{1}{4} A_{4 e}^{-2}+\frac{1}{4} \cos 2 \theta\left(2 A_{6 e}+12 A_{5 e} r^{2}+6 A_{8 e} r^{-4}\right)+\frac{1}{2} A_{3 e} \ln r \\ \tau_{r \theta}^{e} & =\sin 2 \theta\left(\frac{1}{2} A_{6 e}+\frac{3}{2} A_{5 e} r^{2}-\frac{3}{2} A_{8 e} r^{-4}-\frac{1}{2} A_{7 e} r^{-2}\right)\end{array}\right\}$

Formula (5) at its external diameter $r=b$ is still valid. According to the formula (11), due to the interaction in elastic and plastic region, new undetermined parameters $A l e \sim A 8 e$ can be solved and written in matrix form (21). and $(1-b)$,

$$
K\left[\sigma_{r}^{p}\left(P_{i}, P_{z}\right)-\sigma_{1}^{e}\left(P_{u}, P_{z}\right)\right]+B+\sigma_{\theta}^{e}\left(P_{i}, P_{z}\right)-\sigma_{2}^{p}\left(P_{u}, P_{z}\right)=0
$$

$\left(\begin{array}{l}A_{1 e} \\ A_{3 e} \\ A_{4 e} \\ A_{5 e} \\ A_{6 e} \\ A_{7 e} \\ A_{8 e}\end{array}\right)=\left(\begin{array}{ccccccc}\frac{1}{2} & \frac{1+2 L n \rho}{4} & \frac{\rho^{-2}}{4} & 0 & -\frac{\cos 2 \theta}{2} & -\rho^{-2} \cos 2 \theta & -\frac{3 \rho^{-4} \cos 2 \theta}{2} \\ \frac{1}{2} & \frac{3+2 L n \rho}{4} & \frac{-\rho^{-2}}{4} & 3 \rho^{2} \cos 2 \theta & \frac{\cos 2 \theta}{2} & 0 & \frac{3 \rho^{-4} \cos 2 \theta}{2} \\ 0 & 0 & 0 & \frac{3 \rho^{2}}{2} & \frac{1}{2} & -\frac{\rho^{-2}}{2} & -\frac{3 \rho^{-4}}{2} \\ \frac{1}{2} & \frac{1+2 L n b}{4} & \frac{b^{-2}}{4} & 0 & -\frac{1}{2} & -b^{-2} & -\frac{3 b^{-4}}{2} \\ \frac{1}{2} & \frac{3+2 L n b}{4} & \frac{-b^{-2}}{4} & 3 b^{2} & \frac{1}{2} & 0 & \frac{3 b^{-4}}{2} \\ \frac{1}{2} & \frac{1+2 L n b}{4} & \frac{b^{-2}}{4} & 0 & \frac{1}{2} & b^{-2} & \frac{3 b^{-4}}{2} \\ \frac{1}{2} & \frac{3+2 L n b}{4} & \frac{-b^{-2}}{4} & -3 b^{2} & -\frac{1}{2} & 0 & -\frac{3 b^{-4}}{2}\end{array}\right)\left(\begin{array}{c}P_{i} \\ 0 \\ 0 \\ P_{Z} \\ 0 \\ \lambda P_{Z} \\ 0\end{array}\right)$

By analogy with (11), (12), (13), (14), and (15), the stress distribution $\sigma l e(P i, P z), \sigma 2 e(P i, P z)$ in the elastic region can be obtained.
The above formula (22) shows that the unloading pressure $P_{u}$ (the reverse yielding pressure) changes with the internal pressure $P_{i}$. This means that it is not a constant. 
If the internal pressure $P_{i}$ changes in $0 \sim P_{\max }$ periodically, that is $P_{\max }=P_{u=} P_{i}$, implicit equation of $P_{\max }$ can be got according to the formula (24).

$K\left[\sigma_{p r}\left(P_{\max }, P_{z}\right)-\sigma_{1}^{e}\left(P_{\max }, P_{z}\right)\right]+B+\sigma_{p \theta}\left(P_{\max }, P_{z}\right)-\sigma_{2}^{p}\left(P_{\max }, P_{z}\right)=0$

Obviously When $\lambda=1$, the asymmetric problem will degenerate into a symmetric one and the formula (22) can be simplified. We can get explicit equation of $P_{\max }$,

$P_{\max }=\left(\frac{a^{2}+b^{2}}{b^{2}-a^{2}}+\frac{1}{K}\right)^{-1}\left[\frac{2 P_{z}}{1-\left(\frac{a}{b}\right)^{2}}-\frac{B(K+1)}{K}\right]$

As a result of the strain compatibility between casing and cement, we can convert Pmax into the maximum allowable fracturing pressure in the casing.

\section{Sensitivity analysis}

According to the mechanical test results of cement with the same formula system (Zhou et al., 2019), the values of cohesion and internal friction angle can be $7 \mathrm{MPa}$ and $30^{\circ}$, respectively. Due to the centrosymmetry of the sheath shape, $\lambda$ ranging from 0 to 1 can meet all the in-situ stresses conditions with different proportions. What's more, according to worldwide situ stress data (Hoek and Brown, 1980), with the increase of depth, in some extreme areas, the ratio of in-situ

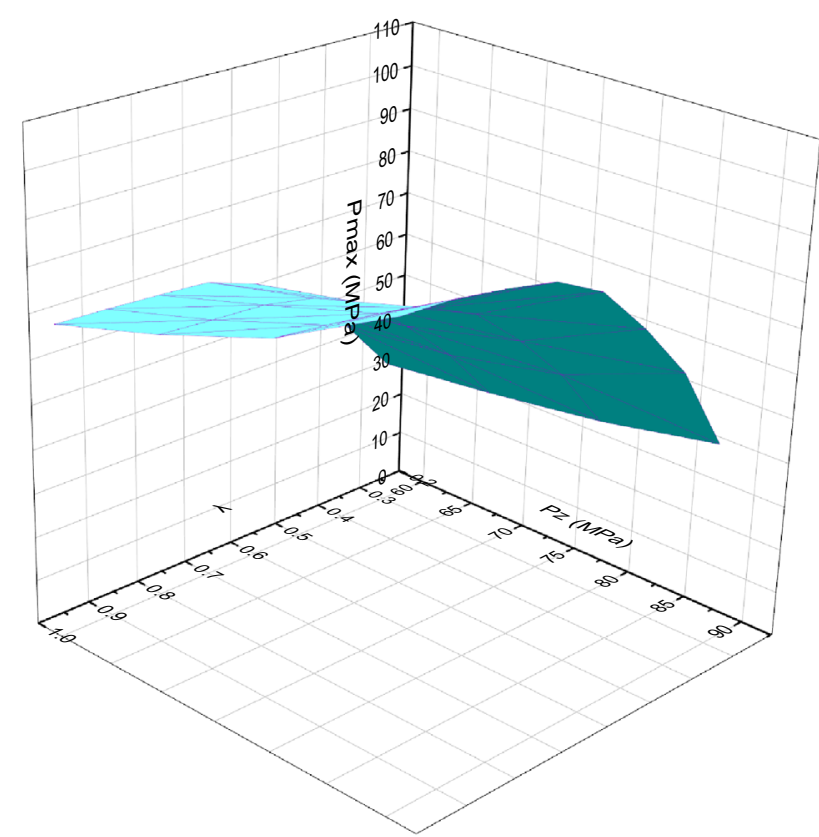

Fig. 4 The Spatial surface formed by $P_{\max }, P z$, and $\lambda$ coordinates

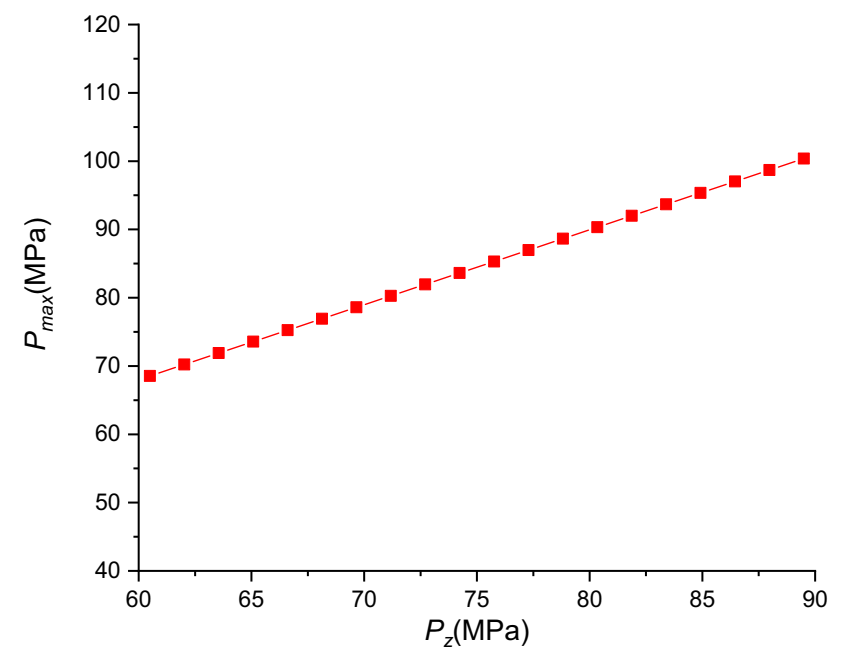

Fig. 5 The relationship between $P_{z}$ and $P_{\max }$

stress in two directions can approach 0.3. Thus, the issue will be discussed in the range where $\lambda$ is from 0.3 to 1 . When the depth is $3000-4500 \mathrm{~m}$, the value of the external pressure $P_{Z}$ can reach $60 \mathrm{MPa} \sim 90 \mathrm{MPa}$ (about $2 \mathrm{MPa} / 100 \mathrm{~m}$ ) considering the engineering practice. By substituting the parameters above into Eq. (24), the effect of asymmetric load on integrity of cement sheath can be obtained.

As shown in Fig. 4, the maximum cyclic loading $P_{\max }$ is related to $P_{z}$ and $\lambda . P_{\max }$ is the function of $P_{z}$ and $\lambda$, and the three form a surface in the coordinate space. Any point satisfying Eq. (24) is on the surface.

$P_{z}$ reflects the mechanical effect of the downhole environment on the cement sheath. Generally, it will grow with the increase of depth. That is, external pressure plays a positive role in the deformation control of the sheath. According to

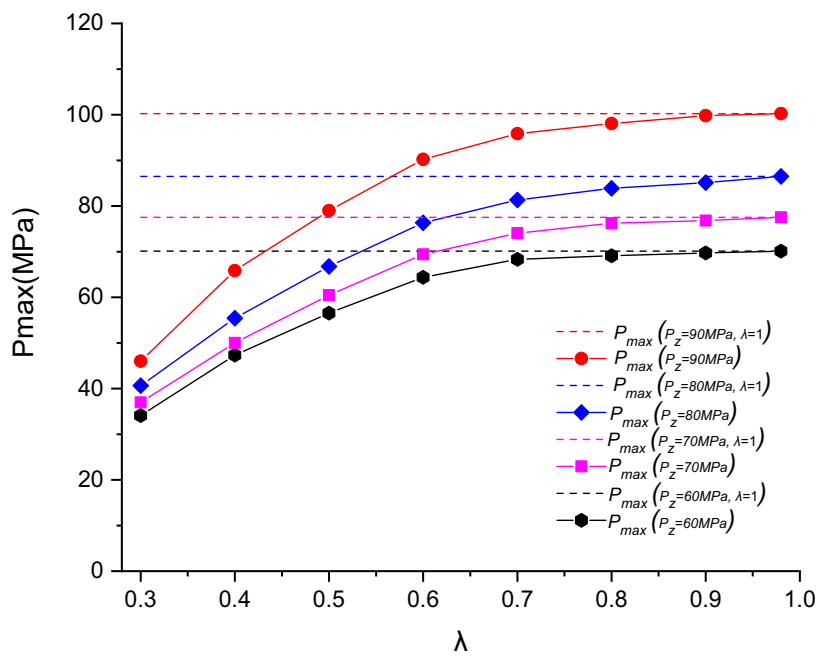

Fig. 6 The relationship between $P_{z}$ and $\lambda$ 
the formula (24), the relationship between $P_{\max }$ and $P_{z}$ can be obtained. As Fig. 5 shows, $P_{\max }$ and $P_{z}$ are linear relations, and the former rises with the increase of the latter. When $P_{z}$ increases from 60 to $90 \mathrm{MPa}$, the $P_{\max }$ rises from 68.53 to $100.36 \mathrm{MPa}$, growing by $46.44 \%$.

The smaller $\lambda$ means the greater the asymmetry. The relationship between $P_{\max }$ and $\lambda$ can be obtained according to formula (24) similarly. Multiple curves of $P_{\max }$ versus $\lambda$ can be drawn by selecting different external pressure $P_{\mathrm{z}}$ in Fig. 6. As mentioned earlier, the external pressure is positively correlated with $P_{\max }$, and the curve corresponding to large external pressure is higher. Obviously, asymmetric stress distribution has an important influence on the bearing capacity of the sheath. When tectonic stress field around the wellbore is abnormal, especially with faults, $\lambda$ will be small. If $\lambda=0.3$ and $P_{\mathrm{z}}=90 \mathrm{MPa}$, the $P_{\max }$ decreases from 100.23 to $46.94 \mathrm{MPa}$ by $53.2 \%$. When $\lambda$ ranges from 0.3 to 1 , with the interval of 0.1 , and the attenuation are $53.2 \%$, $34.3 \%, 21.2 \%, 9.9 \%, 4.37 \%, 2.17 \%$ and $0.43 \%$ successively. Therefore, with the growth of $\lambda$, the stress distribution of the sheath tends to be more symmetrical, and the negative influence of stress asymmetry abates gradually. When $\lambda=0.3$ and $P_{\mathrm{Z}}$ ranges from 60 to $90 \mathrm{MPa}$, the bearing capacity is $48.6 \%$, $47.7 \%, 46.9 \%, 45.9 \%$ of the symmetry. In addition, with the increase of external pressure $P_{Z}$, curves become sparse, that means high $P_{z}$ is beneficial to reduce the negative impact of the asymmetry. With the growth of $\lambda$, the benefit tends to be strengthened.

\section{An engineering example}

The $\mathrm{X}$ well in Fuling, Chongqing, is a pilot hole (vertical well) and horizontal well, with a ground elevation of $734 \mathrm{~m}$. The target layer is the upper Ordovician Wufeng Formation to the lower Silurian Longmaxi Formation shale gas reservoir. After inspection, the cementing quality of each casing and well section is qualified. The shale gas well completed a total of 27 fracturing operations. According to the fracturing design data, the Elastic modulus, Poisson's ratio, cohesion, and internal friction angle are $7 \mathrm{GPa}, 0.15,7 \mathrm{MPa}$, and $27.5^{\circ}$.

On the basis of microseismic monitoring data, it is found that there are faults, leading to the asymmetry of external stress to a certain extent, near segments 6th and 12th, whose $\lambda$ are 0.78 and 0.89 . The 6 th and 12 th segment practical fracturing value are 100.24 MPa and 105.93 MPa greater than the maximum cyclic load of $87.72 \mathrm{MPa}$ and $99.67 \mathrm{MPa}$, exceeding the theoretical values by $14.24 \%$ and $6.28 \%$. Therefore, in the 6th and 12th fracturing sections, it is very likely that after multiple fracturing, the excessive pressures caused the damage of the sheath.
According to field observation, after a series of fracturing work on the $\mathrm{X}$ well, annulus sealing problems happened. Therefore, to a certain extent, it can be verified that the analysis method is valuable for further study.

\section{Conclusions and suggestions}

Based on practical engineering, this paper establishes a mechanical model of the sheath based on the mechanical experiment of cement. Then, the shakedown theory is used for the asymmetric stress problem. The maximum cyclic stress $P_{\max }$ related to $P_{Z}$ and $\lambda$ is discussed.

1) Under high-temperature conditions, the cement stone exhibits obvious plastic flow characteristics and can be regarded as a rational elastic-plastic material. In the process of cyclic loading and unloading, new plastic strains are constantly generated, the width of the "hysteresis loop" of cement sheath is continuously reduced, and the " hysteresis loop " is becoming denser, indicating that the plastic deformation is accumulating in an increasingly slow pace.

2) The external pressure $P_{z}$ plays a positive role in the deformation control of the sheath. $P_{\max }$ and $P_{z}$ are linear relations, and the former rises with the increase of the latter. When the $P_{z}$ increases from 60 to $90 \mathrm{MPa}$, the $P_{\max }$ rises from $68.53 \mathrm{MPa}$ to $100.36 \mathrm{MPa}$, growing by $46.44 \%$.

3) Asymmetric stress distribution has an important influence on the bearing capacity of the sheath. If $\lambda=0.3$, the $P_{\max }$ tends to decrease nearly by $50 \%$. Therefore, with the growth of $\lambda$, the stress distribution of the sheath tends to be more symmetrical, and the negative influence of stress asymmetry relieves gradually. In addition, the higher the $P_{Z}$, the sparse the curves. That means high external pressure is beneficial to reduce the negative impact of the asymmetry. And with the growth of $\lambda$, the benefit tends to strengthen.

4) In engineering practice, if the stress distribution is relatively uniform around wellhole, the bad effect on the sheath can be neglected. However, if the geology around wellhole is complex, especially with faults and large beddings, the stress distribution will suddenly change and present strong asymmetry. Therefore, some steps, such as punching country rock, must be taken to reduce stress concentration.

Funding This work was sponsored by the"National Natural Science Foundation of China" (52104010), and the"Sinopec Science and Technology Department Project" (P21056). 


\section{Declarations}

Conflict of interest The authors declared that they have no conflicts of interest in this work. We declare that we do not have any commercial or associative interest that represents a conflict of interest in connection with the work submitted.

Ethical Approval We certify that this manuscript is original and has not been published and will not be submitted elsewhere for publication while being considered by Journal of Petroleum Exploration and Production Technology. And the study is not split up into several parts to increase the quantity of submissions and submitted to various journals or to one journal over time. No data have been fabricated or manipulated (including images) to support the conclusions. No data, text, or theories by others are presented as if they were our own.

The submission has been received explicitly from all co-authors. And authors whose names appear on the submission have contributed sufficiently to the scientific work and therefore share collective responsibility and accountability for the result.

Open Access This article is licensed under a Creative Commons Attribution 4.0 International License, which permits use, sharing, adaptation, distribution and reproduction in any medium or format, as long as you give appropriate credit to the original author(s) and the source, provide a link to the Creative Commons licence, and indicate if changes were made. The images or other third party material in this article are included in the article's Creative Commons licence, unless indicated otherwise in a credit line to the material. If material is not included in the article's Creative Commons licence and your intended use is not permitted by statutory regulation or exceeds the permitted use, you will need to obtain permission directly from the copyright holder. To view a copy of this licence, visit http://creativecommons.org/licenses/by/4.0/.

\section{References}

Al Ramis H, Teodoriu C, Bello O, al Marhoon, Z., (2020) High definition optical method for evaluation of casing - Cement microannulus (CCMA). J Petrol Sci Eng. https://doi.org/10.1016/j.petrol. 2020.107719

Gholami R, Aadnoy B, Fakhari N (2016) A thermo-poroelastic analytical approach to evaluate cement sheath integrity in deep vertical wells. J Petrol Sci Eng 147:536-546. https://doi.org/10.1016/j.petrol.2016.09.024

Gu J, Zhong P, Shao C, Bai S, Zhang H, Li K (2012) Effect of interface defects on shear strength and fluid channeling at cement-interlayer interface. J Petrol Sci Eng 100:117-122. https://doi.org/10.1016/j. petrol.2012.11.021

Guo B, Shan L, Jiang S, Li G, Lee J (2018) The maximum permissible fracturing pressure in shale gas wells for wellbore cement sheath integrity. Journal of Natural Gas Science and Engineering 56:324-332. https://doi.org/10.1016/j.jngse.2018.06.012

Hoek E, Brown ET (1980) Underground Excavations in Rock. Institute of Mining and Metallurgy, London, p 527

Jun G, Wenzheng Q (2010) Experiments on integrated solidification and cementation of the cement-formation interface based on mud cake to agglomerated cake (MTA) method. Pet Explor Devel 37(2):226-231

Lavrov A, Gawel K, Stroisz A, Torsæter M, Bakheim S (2017) Failure modes in three-point bending tests of cement-steel, cement-cement and cement-sandstone bi-material beams. Constr Build Mater 152:880-886. https://doi.org/10.1016/j.conbuildmat.2017.07.017

Li Z, Sun J, Luo PY, Lin L, Deng Z, Guo X (2017) Research on the law of mechanical damage-induced deformation of cement sheaths of a gas storage well. Journal of Natural Gas Science and Engineering 43:48-57. https://doi.org/10.1016/j.jngse.2017.03.026
Liu K, Gao D, Taleghani AD (2018) Analysis on integrity of cement sheath in the vertical section of wells during hydraulic fracturing. J Petrol Sci Eng 168:370-379. https://doi.org/10.1016/j.petrol. 2018.05.016

Neal B G . The plastic methods of structural analysis. Chapman And Hall Ltd, 1956.

Shen Y, Wei X, Yang G, Wang Y, Jia H, Zhang Huan, Zhang Huimei (2020) Freeze-thaw degradation model and experimental analysis of rock-concrete interface bond strength. Yanshilixue Yu Gongcheng Xuebao/Chinese. J Rock Mech Eng 39:480-490

Sun J-S, Lu W-B (2007) Analytical elastoplastic solutions to supporting rock masses of circular tunnels under asymmetric load

Welch NJ, Frash LP, Harp DH, Carey JW (2020) Shear strength and permeability of the cement-casing interface. Int J Greenhouse Gas Control. https://doi.org/10.1016/j.ijggc.2020.102977

Xi Y, Li J, Fan L, Wang W, Zha C (2021) Mechanism and numerical simulation of a new device of bypass cementing device for controlling casing shear deformation induced by fault slipping. J Petrol Sci Eng. https://doi.org/10.1016/j.petrol.2020.107820

$\mathrm{Xu} \mathrm{H}$, Ma T, Peng N, Yang B (2018a) Influences of fracturing fluid injection on mechanical integrity of cement sheath under four failure modes. Energies. https://doi.org/10.3390/en11123534

Xu H, Peng N, Ma T, Yang B (2018b) Investigation of thermal stress of cement sheath for geothermal wells during fracturing. Energies. https://doi.org/10.3390/en11102581

Yan W, Zou L, Li H, Deng J, Ge H, Wang H (2017) Investigation of casing deformation during hydraulic fracturing in high geo-stress shale gas play. J Petrol Sci Eng. https://doi.org/10.1016/j.petrol. 2016.11.007

Yang H, Bu Y, Guo S, Liu H, Du J, Cao XC (2021) Effects of in-situ stress and elastic parameters of cement sheath in salt rock formation of underground gas storage on seal integrity of cement sheath. Eng Fail Anal. https://doi.org/10.1016/j.engfailanal.2021.105258

Yarveicy, H., Habibi, A., Pegov, S., Zolfaghari, A., \& Dehghanpour, H. (2018). SPE-189829-MS Enhancing Oil Recovery by Adding Surfactants in Fracturing Water: A Montney Case Study.

Yuxiang L, Yongjiu P, Quankun L, Yi W (2012) Approximate calculation for plastic zone boundary of circularcavity under the asymmetric load. Highway Engineering 37:128-131

Zarka J ,Casier J. Cyclic loading on elastic-plastic structures. Mechanics Today. S. Nemat Nasser Ed. Pergaman Press, Oxford,1979,93 198.

Zeng Y, Liu R, Li X, Zhou S, Tao Q, Lu P (2019) Cement sheath sealing integrity evaluation under cyclic loading using large-scale sealing evaluation equipment for complex subsurface settings. $\mathbf{J}$ Petrol Sci Eng 176:811-820. https://doi.org/10.1016/j.petrol.2019. 02.014

Zhang Z, Wang H (2017) Effect of thermal expansion annulus pressure on cement sheath mechanical integrity in HPHT gas wells. Appl Therm Eng 118:600-611. https://doi.org/10.1016/j.appltherma leng.2017.02.075

Zhang H, Shen R, Yuan G, Ba Z, Hu Y (2017) Cement sheath integrity analysis of underground gas storage well based on elastoplastic theory. J Petrol Sci Eng 159:818-829. https://doi.org/10.1016/j. petrol.2017.10.012

Zhao C, Li J, Liu G, Zhang X (2019) Analysis of the influence of cement sheath failure on sustained casing pressure in shale gas wells. Journal of Natural Gas Science and Engineering 66:244254. https://doi.org/10.1016/j.jngse.2019.04.003

Zhou S, Liu R, Zeng H, Zeng Y, Zhang L, Zhang J, Li X (2019) Mechanical characteristics of well cement under cyclic loading and its influence on the integrity of shale gas wellbores. Fuel 250:132-143. https://doi.org/10.1016/j.fuel.2019.03.131

Publisher's Note Springer Nature remains neutral with regard to jurisdictional claims in published maps and institutional affiliations. 